\title{
Solid-state power converter repeatability analysis
}

Anthony Dal Gobbo, Davide Aguglia

CERN, Geneva, Switzerland,

Keywords: «Switched-mode power supply», «Pulsed power converter», «Semiconductor device».

\begin{abstract}
This paper presents a method for evaluating power converter repeatability. The focus is on solid-state switch mode power converter for which the most problematic non-repeatability sources are the jitter of the drivers and of the switches leading to output voltage pulses bad repeatability. Both driver and switch turn-on and turn-off delay dispersion have been measured. These measurements confirm that the delay is Gaussian distributed and that the repeatability prediction method is valid.
\end{abstract}

Presented at: EPE 2015, 7-10 September 2015, Geneva, Switzerland

Geneva, Switzerland

October, 2015 


\title{
Solid-state power converter repeatability analysis
}

\author{
Anthony Dal Gobbo and Davide Aguglia \\ CERN-European Organization for Nuclear Research, Technology Department, Electrical \\ Power Converter group \\ 1211 Geneva 23, Switzerland \\ Tel.: $+41 /(0)-227662052$ \\ E-Mails: anthony.dal.gobbo@cern.ch,davide.aguglia@cern.ch \\ URL: www.cern.ch
}

\section{Keywords}

«Switched-mode power supply», «Pulsed power converter», «Semiconductor device».

\section{Acknowledgment}

The Authors would like to thank Mr. Carlo Baccigalupi, CERN Ph.D. student affiliated to the University of Calabria (IT), for his contribution in the setting-up of the high precision measurement system.

\begin{abstract}
This paper presents a method for evaluating power converter repeatability. The focus is on solid-state switch mode power converter for which the most problematic non-repeatability sources are the jitter of the drivers and of the switches leading to output voltage pulses bad repeatability. Both driver and switch turn-on and turn-off delay dispersion have been measured. These measurements confirm that the delay is Gaussian distributed and that the repeatability prediction method is valid.
\end{abstract}

\section{Introduction}

The Compact Linear Collider (CLIC) is an electron-positron linear collider under study at CERN [1]. The specification in terms of Radio Frequency (RF) repeatability from the klystrons used to accelerate the drive beam is very tight. The RF quality is linked both to the High Voltage produced by the Klystron modulator and the Low Level RF control [2]. The LLRF is capable of correcting nonrepeatability below a few tens of $\mathrm{kHz}$ depending on the klystron and the RF feedback. This paper gives a clear definition of voltage pulse repeatability and aims to provide an efficient simulation method for repeatability evaluation of switch mode power converter. The only source of nonrepeatability considered are the driver and switch jitter and their jitter is assumed to be Gaussian distributed. The measurement of turn-on and turn-off delay dispersion (jitter) have confirmed this assumption.

Output voltage repeatability evaluations have been performed both using the simulation model of a simple buck converter, and on the equivalent real system we have developed. The comparison of simulation results with experimental measurement results allows to validate the method and specific assumptions.

\section{Source of non-repeatability in switch mode power converter}

Modern klystron modulators are based on semiconductor power switches. The advantages are the possibility to operate the power converter in feedback loop, and to easily control the pulse length and the voltage level. Basically, a constant DC voltage is chopped by one or several switches and then smooth by a filter designed such that its bandwidth is a trade-off between the pulse rise time and the remaining output ripple [3]. When operating a switch mode power converter, one expects the driving 
switch command to be nicely followed by the switch itself. Unfortunately, random noise phenomena such as thermal noise and shot noise occurs in semiconductors, therefore switch drivers and switches are affected by a random turn-on and turn-off time jitter [4]. These jitters lead to the impossibility to perfectly command a switch. As a consequence the output voltage of such power converters presents a stochastic behaviour with respect to the switch control signal. Figure 1 illustrates a simplified visualization of the switch jitter impact on turn-on and turn-off time lags with respect to the switch control (from a driver).

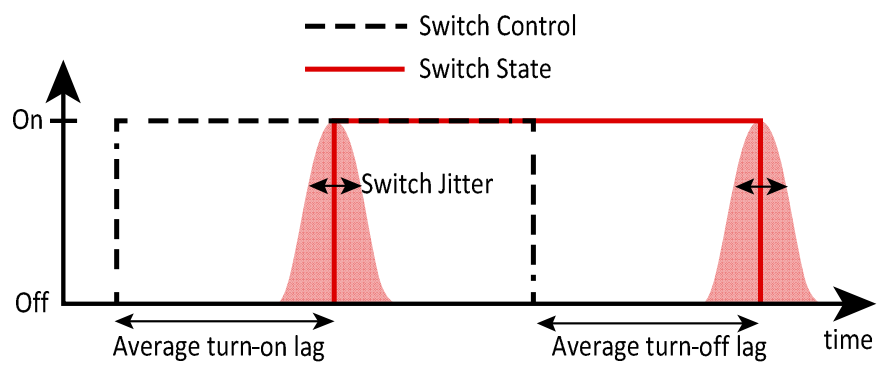

Fig1: Schematic of the switch jitter: the dashed line represents the switch control signal and the red line the switch most probable actual state.

\section{Pulse repeatability definition}

Supposing a set of repetitive pulses, derived from measurement or numerical simulations, one can obtain the matrix $\mathrm{V}$ in equation (1). The matrix represents the voltage of a set of $\mathrm{N}$ pulses of length $\mathrm{t}_{\mathrm{f}}$, $\mathrm{V}_{\mathrm{i}}\left(\mathrm{t}_{\mathrm{j}}\right)$ being the voltage of the pulse $\mathrm{i}$ at the time $\mathrm{t}_{\mathrm{j}}$.

$$
V=\left[\begin{array}{cccc}
V_{1}\left(t_{1}\right) & V_{1}\left(t_{2}\right) & \ldots & V_{1}\left(t_{f}\right) \\
V_{2}\left(t_{1}\right) & V_{2}\left(t_{2}\right) & \ldots & V_{2}\left(t_{f}\right) \\
\ldots & \ldots & \ldots & \ldots \\
V_{N}\left(t_{1}\right) & V_{N}\left(t_{2}\right) & \ldots & V_{N}\left(t_{f}\right)
\end{array}\right]
$$

Since switch random jitter, and consequently the converter repeatability, is a stochastic phenomenon, there is no correlation between any pulses of a given set. Therefore, a set of $\mathrm{N}$ voltage samples for a given time $t_{j}$ (one column of the $V$ matrix) can be seen as the realization of the random variable $\mathrm{X}_{\mathrm{tj}}$ of standard deviation $\sigma_{\mathrm{Xtj}}$. In practice, one can calculate the standard deviation $\sigma_{\mathrm{tj}}$ of $\mathrm{N}$ voltage samples and approach $\sigma_{\mathrm{Xtj}}$ by increasing the number of samples $\mathrm{N}$. The higher the considered number of pulses and time samples, the higher the accuracy in estimating the repeatability.

\section{Tolerance interval}

The switch jitter induces Gaussian distributed voltage dispersion at each time $t_{j}$ (from $t_{1}$ to $t_{f}$ ) within the pulses as shown in Figure 5. A tolerance interval $\alpha$ which defines the probability for pulses to meet the repeatability specification such that the pulse repeatability at the time $t_{j}$ is $P R_{t j}=\alpha \cdot \sigma_{t j}$ must be introduced. Table 1 shows the percentage of pulses which meet the specification for different values of $\alpha$.

Table I: Probability to meet the spec. vs. tolerance interval

\begin{tabular}{|c|c|}
\hline$\alpha$ & Probability to meet the spec. at time $\mathrm{t}_{\mathrm{j}}$ \\
\hline 2 & $\sim 68.2 \%$ \\
\hline 4 & $\sim 95.4 \%$ \\
\hline 6 & $\sim 99.7 \%$ \\
\hline
\end{tabular}

\section{Repeatability evaluation methods}

At each time $t_{j}$, the standard deviation $\sigma_{\mathrm{tj}}$ of a set of $\mathrm{X}_{\mathrm{tj}}$ realization is derived from the columns of matrix $V$. The PR for each time $\mathrm{t}_{\mathrm{j}}$ is obtained by multiplying each $\sigma_{\mathrm{tj}}$ by the tolerance interval $\alpha$. 
Finally, several pieces of information can be extracted:

- the PR as a function of time and the PR frequency spectrum by performing Fourier transform,

- the peak PR by taking the greatest $\sigma_{t j}$ obtained (worst case).

The absolute PR is the maximum accepted voltage difference between any two pulses but it might be exceeded a certain percentage of time, defined by the tolerance interval.

\section{Validation of the repeatability evaluation method}

In order to validate the repeatability calculation method, the jitter dispersion hypothesis has been verified in practice to ensure that the switch jitter and the driver jitter are Gaussian distributed. Then, the repeatability evaluation method has been performed both numerically and experimentally on the very same power converter topology. Thus, the simulation model developed was the model of the $1 \mathrm{MW}$ test bed used to carry out experimental measurements. This power converter is described in the next section, followed by the experimental measurement of the switch and driver jitter and the comparison of simulation and experimental result.

\section{Studied power converter description}

The power converter studied consists of a buck converter with an additional parallel damping branch as shown in Figure 2. The main component values are listed in Table III. The capacitor charger is rated at $10 \mathrm{~kW}$ and can deliver a current up to 10A. The IGBT implemented is an ABB HiPak IGBT Module (5SNA $1600 \mathrm{~N} 170100$ ) with $1700 \mathrm{~V}$ maximum collector-emitter voltage and 1600A DC collector current. The Diode is a fast recovery diode module Dynex DFM1200FXM18-A00 with 1800V repetitive peak reverse voltage and 2400A maximum forward current nominal current. The driver is a Concept 1SP0635V2M1-17 with optic-fiber interface.

The system has been though and dimensioned to work at $10 \mathrm{kHz}$ of switching frequency with a ripple greater than $1 \%$ for a duty cycle of 0.5 .
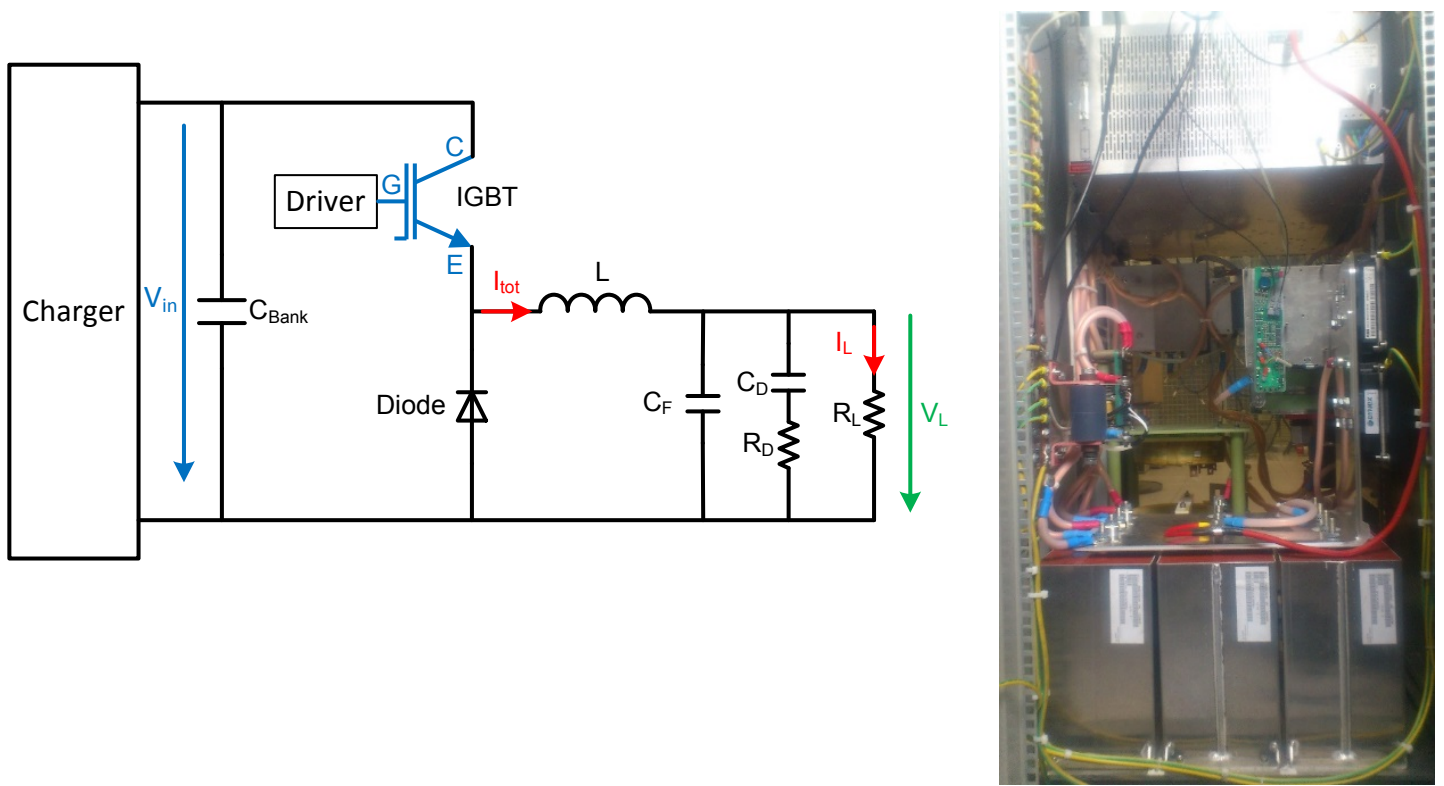

Fig 2: Power converter schematic (left) and test bed picture (right).

Table III: Test bed component characteristics

\begin{tabular}{|c|c|c|c|}
\hline Bank capacitor & $\mathrm{C}_{\text {Bank }}$ & 9 & $\mathrm{mF}$ \\
\hline Inductance & $\mathrm{L}$ & 150 & $\mathrm{mF}$ \\
\hline Filter Capacitance & $\mathrm{C}_{\mathrm{F}}$ & 86 & $\mu \mathrm{F}$ \\
\hline Damping Resistor & $\mathrm{R}_{\mathrm{D}}$ & 0.1 & $\Omega$ \\
\hline
\end{tabular}




\begin{tabular}{|l|l|l|l|} 
Load Resistor & $\mathrm{R}_{\mathrm{L}}$ & 1 & $\Omega$ \\
\hline
\end{tabular}

The measurement and acquisition system have been designed at CERN [5] and accurately characterized [6].

\section{Experimental pulse measurement technique}

The pulses voltage has been obtained by measuring the current in the $1 \Omega$ load resistor with a current transformer (CT). To benefit of a higher digital resolution in the flat top range voltages, only the flattop has been acquired. To do so, the output of a high repeatability reference DC voltage generator set to the flat-top mean voltage has been subtracted from the CT measurement.

\section{PWM sequence pre-determination for generating the ideal pulse}

The power converter is operated in open-loop, the switching command sent to the driver had to be predetermined such to obtain a voltage pulse to the load with no overshoot and a stabilized flat-top. In order to obtain the ideal command sequence, a control loop has been added to the simulated model of the test bed buck converter. An experimental parametric identification has been carried out in order to obtain good parameters in the simulation process. The control loop has been tuned so as to obtain the desired output voltage pulse shape and the corresponding driver command has been acquired. This signal was the one applied both to the simulation model and to the real system via an Arbitrary Function Generator.

\section{Jitter injection}

The test bed presents the possibility to insert a known Gaussian time jitter in the driver ideal command which artificially increases the intrinsic jitter of the IGBT and the driver themselves (Figure 3).

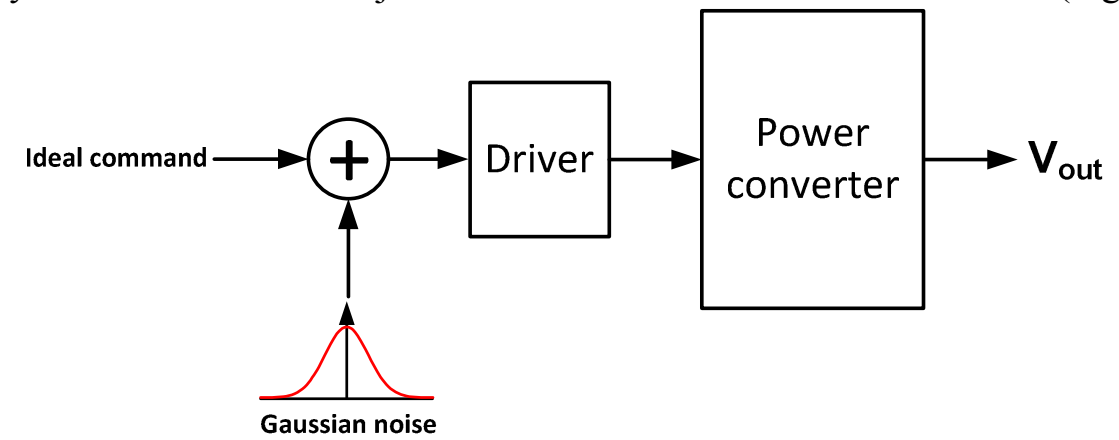

Fig 3: Jitter injection schematic. A Gaussian noise (with settable standard deviation) is added to the ideal driver input.

The possibility to increase the command chain time jitter allow boosting the non-repeatability of the pulse, therefore it can be measured more easily and more accurately as the voltage measurement precision is limited. Indeed, the pulses non-repeatability must be greater than the measurement repeatability otherwise one cannot access the pulses repeatability which is hidden by the measurement limitation.

\section{Jitter measurement}

The first measurement consists in characterizing the driver and the switch turn-on and turn-off jitter with the aim of verifying their statistic distribution. In order to perform statistical analysis, the driver input voltage, the IGBT gate voltage $\mathrm{V}_{\mathrm{GE}}$ and the voltage at the IGBT power terminals (Vce) have been measured for two hundred IGBT turn-on and two hundred turn-off. Figure 4 shows the driver input, $\mathrm{V}_{\mathrm{GE}}$ and $\mathrm{V}_{\mathrm{CE}}$ as a function of time during one IGBT turn-off as well as the driver input-to- $\mathrm{V}_{\mathrm{GE}}$ delay distribution and the $\mathrm{V}_{\mathrm{GE}}-\mathrm{to}-\mathrm{V}_{\mathrm{CE}}$ delay distribution. 
Delay distributions shows that both the driver and the IGBT jitters are Gaussian distributed and validate the initial assumptions on the jitter statistical description. The driver jitter shows a Gaussian distribution with a standard deviation of $0.6 \pm 0.1 \mathrm{~ns}$ and the IGBT jitter, a standard deviation of $0.8 \pm 0.1 \mathrm{~ns}$. Similar result have been obtained for the IGBT turn-on. This proves that the jitter injection with Gaussian noise make sense and can be used to experimentally magnify the non-repeatability of the pulse voltage.
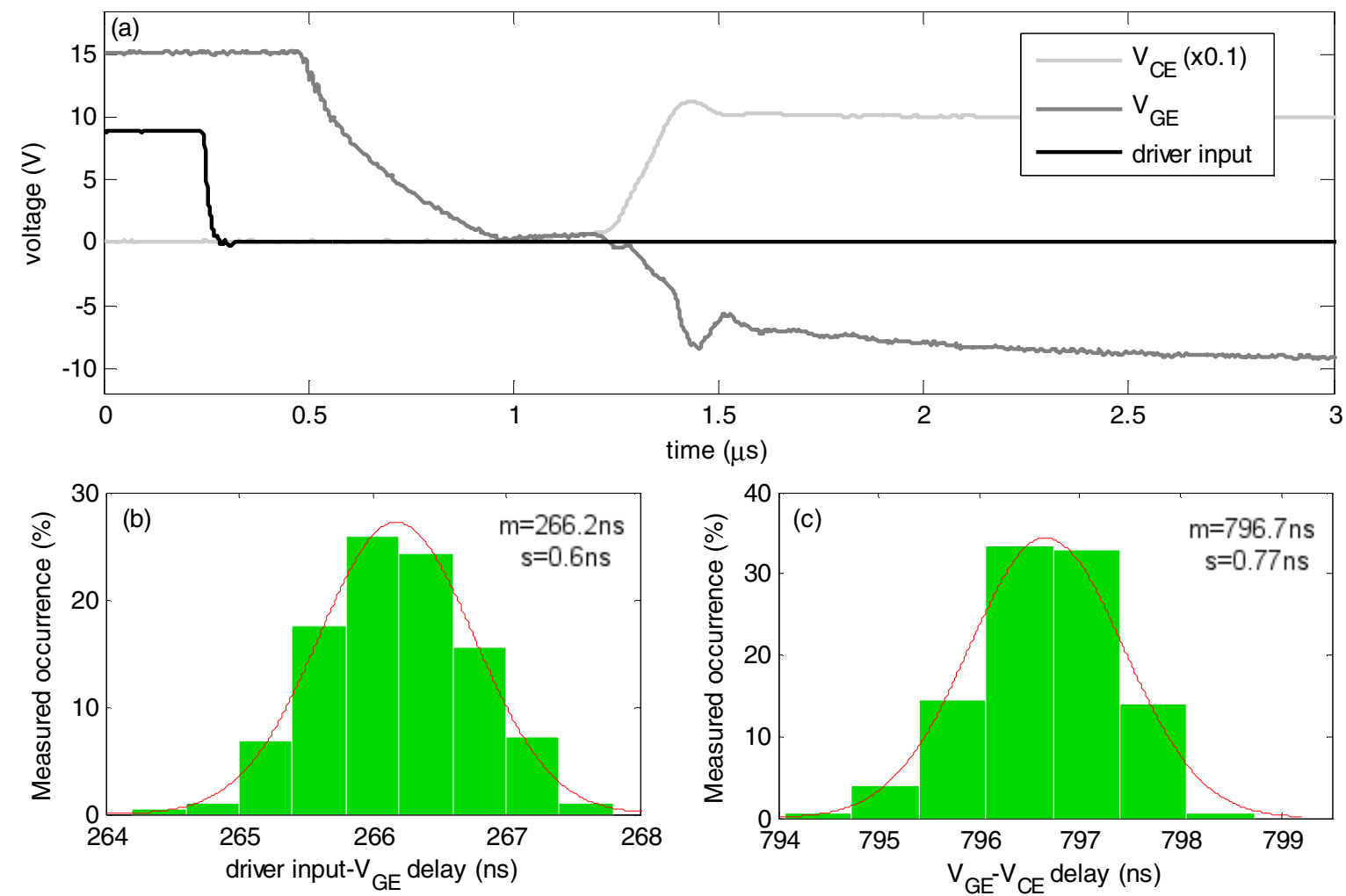

Fig 4: (a) Turn-off waveforms. Driver input, $\mathrm{V}_{\mathrm{GE}}$ and $\mathrm{V}_{\mathrm{CE}}$ vs. time. (b) Driver input-to- $\mathrm{V}_{\mathrm{GE}}$ delay distribution. (c) $\mathrm{V}_{\mathrm{GE}}$-to- $\mathrm{V}_{\mathrm{CE}}$ delay distribution.

\section{Repeatability evaluation by numerical simulation vs. experimental measurement}

This section aims at comparing the repeatability evaluation results from numerical simulations and from experimental measurement in order to validate the numerical method. The very same driver command was used to generate pulses in both cases. This command generates pulses with $50 \%$ duty cycle. The DC bus voltage $\mathrm{V}_{\mathrm{DC}}$ has been set to $100 \mathrm{~V}$. The absolute repeatability was converted in relative repeatability expressed in ppm as follow:

$$
\operatorname{rep}(p p m)=\frac{P R \times 10^{6}}{V_{D C}}=\frac{P R \times 10^{6}}{100}
$$

The tolerance coefficient in the following results has been set to 1 . The pulse repeatability has been measured and simulated for the following switching jitter value: $4 \mathrm{~ns}, 10 \mathrm{~ns}, 15 \mathrm{~ns}$, 20ns, $25 \mathrm{~ns}$, $30 \mathrm{~ns}$ and 40ns. This jitters are the total command jitter that is, the intrinsic jitter of the driver and the switch plus the injected jitter. In practice, the command jitter has been measured with an uncertainty of $\pm 1 \mathrm{~ns}$. 

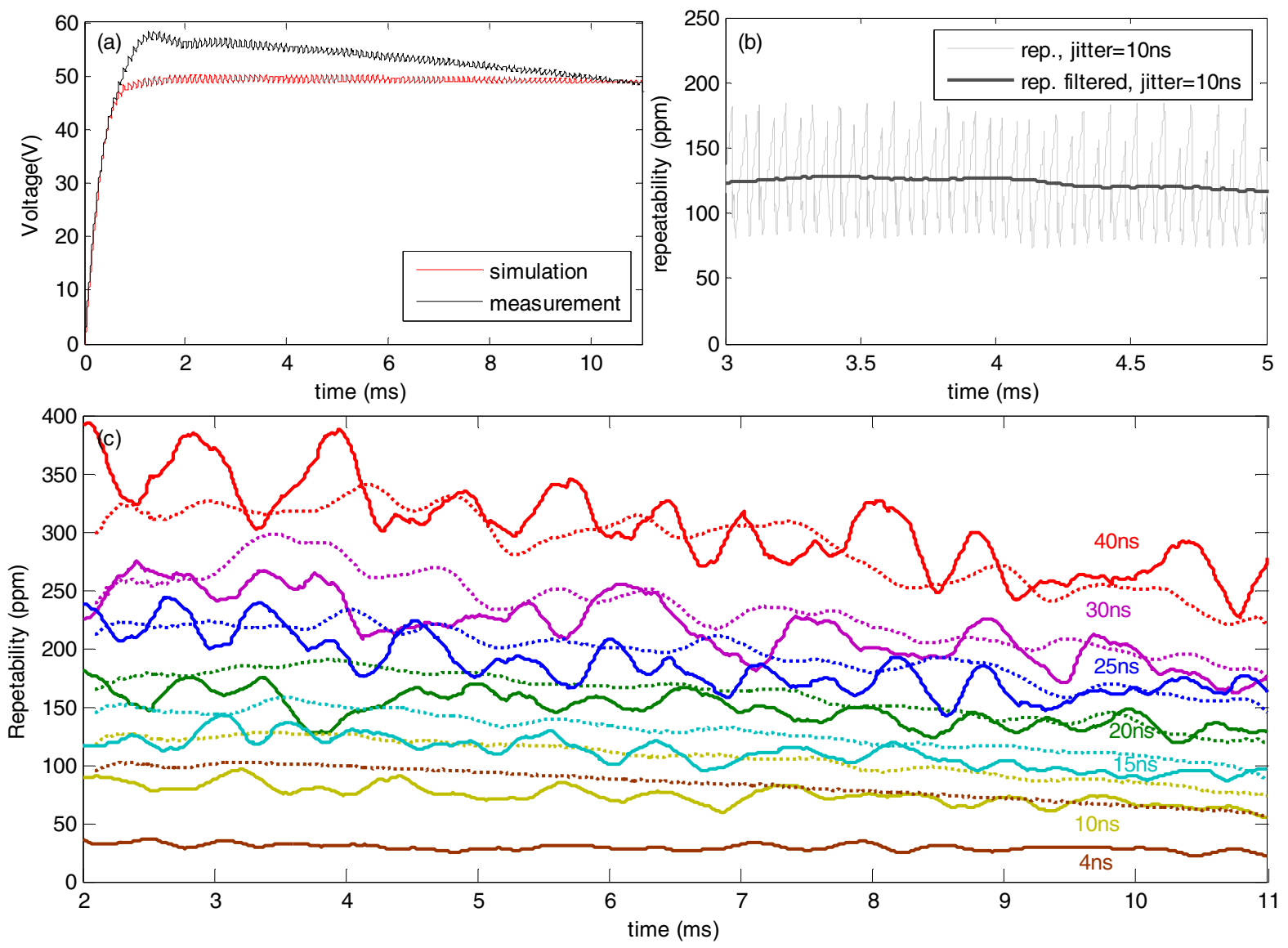

Fig 5: (a) Example of a voltage pulse obtained by simulation and measured on test bed. (b) Zoom on experimental repeatability vs. time for a $10 \mathrm{~ns}$ jitter. In grey, the raw repeatability, in black the repeatability filtered at $10 \mathrm{kHz}$. (c) Numerical (solid lines) and experimental (dashed lines) repeatability vs. time for a jitter of 40ns (red), 30ns (purple), 25ns (blue), 20ns (green), 15ns (cyan), 10ns (yellow) and 4ns (brown).

Figure 5(a) shows the pulse voltage shapes obtained numerically and experimentally. The voltage droop in the experimental pulse is due to the fact that the voltage has been derived by a current measurement through a current transformer CT. It can be noticed that it has no influence on the pulse repeatability as the droop can be considered repeatable. Figure 5(b) illustrates the repeatability obtained experimentally for a $10 \mathrm{~ns}$ jitter. A $20 \mathrm{kHz}$ frequency oscillation can be observed which is the double of the switching frequency. We assume this to be due to a non-perfect synchronization of the pulses starts which would, indeed, generate a non-repeatability maximum at the ripple peaks (minimum and maximum) therefore at twice the switching frequency. In order to get rid of this measurement issue, this frequency and its harmonics has been filtered (black line on figure 5(b)). Figure 5 (c) shows both the numerical and experimental pulse flat-top voltage repeatability as a function of time for jitters up to 40ns.

For high jitter values (above 15ns), experimental repeatability fits particularly well the numerical repeatability whereas a significant discrepancy can be observed for lower jitter values. The lower the jitter, the higher the difference between simulation and measurement.

For comparison purposes, the average of each pulse repeatability as function of time curve has been calculated and represented in Figure 6. As intuitively expected, simulation results show a linear relation between jitter and repeatability. 


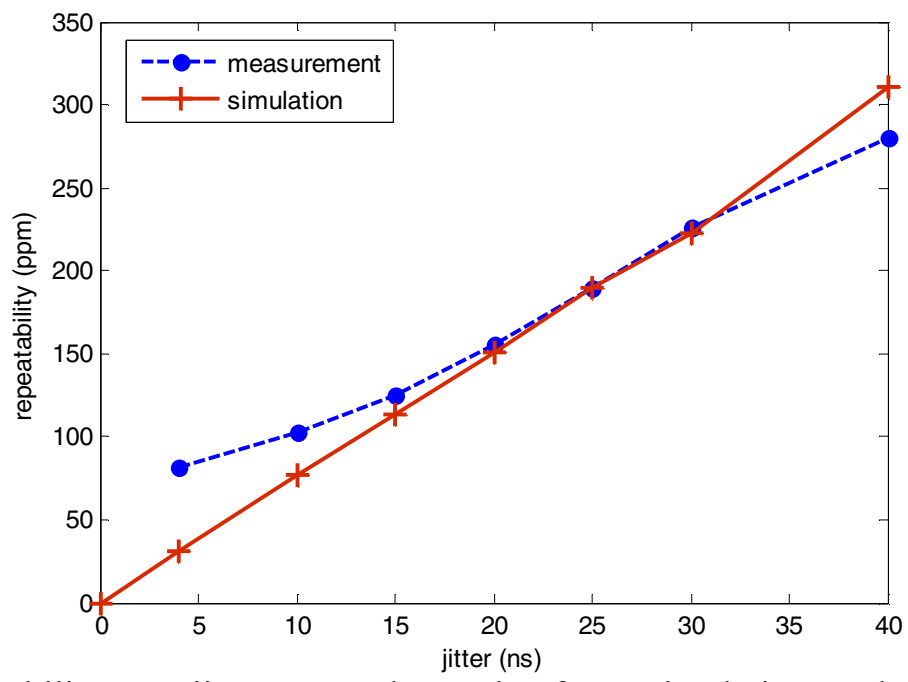

Fig 6: Pulse repeatability vs. jitter. In red, results from simulation and in blues results from measurement.

For low jitter, measurement capacities as well as the other non-repeatability sources (e.g. temperature swings, electromagnetic environment, etc.) are no longer negligible with regards to the nonrepeatability due to the switch jitter. As a consequence, for high jitter value, both experimental and numerical pulse repeatability increases linearly with respect to switch jitter whereas for low jitter values, the experimental repeatability evaluation tend to a minimum limit. With a low jitter IGBT and a low jitter driver we have been able to reach an overall jitter of 4 ns (no jitter injection). The repeatability measured with this jitter was $81 \mathrm{ppm}$ but Figure 6 shows that this value is overestimated as numerical simulation gives a repeatability of $31 \mathrm{ppm}$.

\section{Conclusion}

When the repeatability objective get close or even get below the measurement capability, the measured pulse repeatability is actually a mix of the repeatability due to the driver and the IGBT jitters, the measurement repeatability and the non-repeatability due to the other non-repeatability sources. This study shows that the converter output pulse non-repeatability due to low jitter drivers and switches can be estimated, even with the incapability to measure directly its contribution in pulse voltage repeatability. This is made possible only if the driver and the IGBT jitters are Gaussian distributed. Therefore, one should, first, check if the components in the command chain of his power converter respect this feature. For instance slow digital drivers presents a non-Gaussian jitter distribution, leading to inconsistencies between the theoretical and experimental approach.

\section{References}

[1] CERN - European Organization for Nuclear Research. "The CLIC Programme towards a staged $\mathrm{e}^{+} \mathrm{e}^{-}$Linear $^{-}$ Collider exploring the Terascale", CLIC Conceptual Design Report, 2012.

[2] R. Zeng, D. McGinnis and S. Molloy, "Investigation of Feedback control for klystron ripple", Proceedings of IPAC2012, New Orleans, Louisiana, USA, pp. 1-3.

[3] F. Cabaleiro Magallanes, D. Aguglia, C. A. Martins, and P. Viarouge "Active Damping Filter for High Bandwidth - Low Ripple Pulsed Converters”, 15 ${ }^{\text {th }}$ EPE-PEMC 2012 ECCE Europe, Novi Sad, Serbia, pp. DS2b.15-1 - DS2b.15-7.

[4] Johnnie Hancock, “Jitter-Understanding it, Measuring it, Eliminating It - Part 1: Jitter Fundamentals', High Frequency Electronics, pp 45-46, April 2004.

[5] P. Arpaia, C Baccigalupi, M. Cerqueira Bastos, and M. Martino, "25 ppm Repeatable Measurement of Trapezoidal Pulses with 5 MHz Bandwidth”, Journal of Instrumentation, 9(06): P06002, 2014.

[6] P. Arpaia, C. Baccigalupi, M. Martino "Metrological Characterization of an Ultra-Low Noise Acquisition System for Fast Voltage Pulses Measurements", In IEEE International Instrumentation and Measurement Technology Conference (I2MTC), May 11-14 2015, Pisa, Italy. 